\title{
MULTILAYERED LOW PASS MICROSTRIP FILTER USING CSRR
}

\author{
Prachi Agrawal ${ }^{1}$, Vandana V. Thakare ${ }^{2}$ \\ ${ }^{1}$ Student, Dept. of Electronics and Communication Engineering, Madhav Institute of Technology and Science, Gwalior, \\ India \\ ${ }^{2}$ Associate Professor, Dept. of Electronics and Communication Engineering, Madhav Institute of Technology and Science, \\ Gwalior, India
}

\begin{abstract}
In the present paper a multilayered microstrip low pass filter using complementary split ring resonator is proposed. A design for prominent stop band characteristics with minimized ripples is presented, while maintaining the filter pass-band performance. By properly designing and integrating the complementary split ring resonators with the low pass filter, the proposed structure exhibit superior pass band and stop band characteristics by eliminating unwanted spurious signals. Since the literature is multi-layered, no structure is designed at the ground plane and the problem of distortion of ground plane structure while packaging is resolved. The measured results indicate that the proposed structure achieves significantly improved band characteristics with minimum distortion, when compared with the simulated one.
\end{abstract}

Keywords: Low Pass filter; Multilayered; Metamaterial; Complementary split ring resonator (CSRR) structure $* * *$

\section{INTRODUCTION}

A microwave filter is a two port network used to control the frequency response at a certain point in a microwave system [1]. More specifically a low pass filter is a filter that passes low frequency signals but attenuates signals with frequencies higher than cut off frequency as shown in Fig.1. The paper describes about the design and fabrication of multilayered low pass filter by using microstrip layout which works at $2 \mathrm{GHz}$.

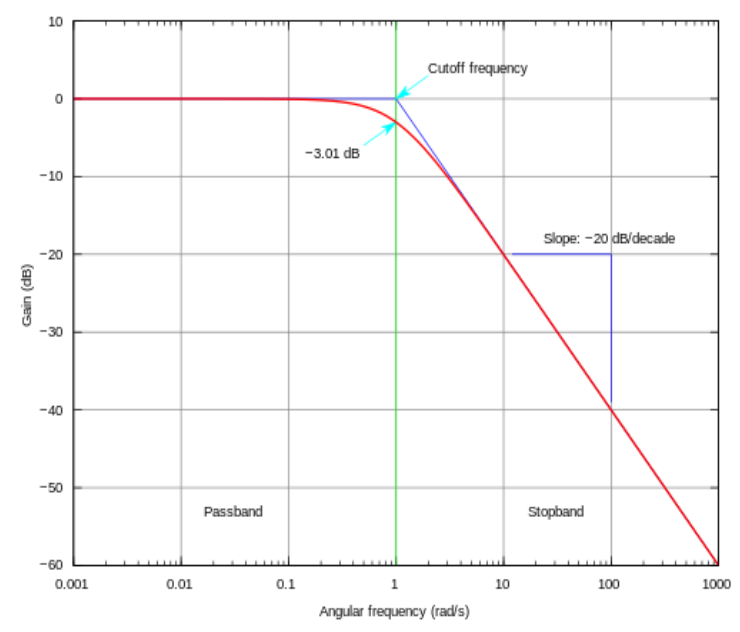

Fig. 1 Response of low pass filter

Recently, there has been a growing interest for the use of metamaterials in the development of compact microwave components constructed using printed circuit board technology. Metamaterials are artificial materials engineered to have properties that may not be found in nature. Metamaterial usually gain its properties from structure rather than composition, using small inhomogeneities to create effective macroscopic behavior. Specifically, the development of Complementary split ring resonators (CSRRs), originally proposed by Pendry et al. [2] was a major breakthrough for implementing left-handed techniques in planar circuit technology. Pendry et al. proposed that the frequency selective property of SRRs was due to the negative effective permeability, produced at resonance. Another interesting particle proposed is the complementary split ring resonator (CSRR).This is a negative image of the SRR and hence is its dual counterpart. The CSRR behaves as an element, which is able to produce a negative effective permittivity at resonance. CSRRs can be etched in the ground plane, beneath the microstrip for stop band filter design and to eliminate the unwanted spurious signals. A general CSRR structure is shown in Fig. 2

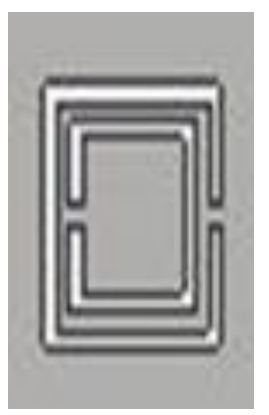

Fig. 2 A general CSRR structure 
Low-pass filters have been studied and exploited extensively as a key block in modern communication systems. A planar microwave filter is widely used in microwave systems because of its easy fabrication. According to microstrip theory, in order to control the characteristic impedance of a microstrip segment, the physical dimensions of the microstrip line are adjusted. The physical dimensions namely length (l) and width (w) depends on substrate thickness (d) and dielectric constant.

The paper describes about the design and fabrication of microwave low pass filter by using micro strip layout which resonates at $2.0 \mathrm{GHz}$. It has been demonstrated that the selectivity of the filter increases and the filter size reduces by the use of multilayer structure. Since the design is multilayered, no structure is designed at the ground plane so the problem of distortion of ground plane structure while packaging and handling is resolved.

\section{FILTER DESIGNING}

The proposed multi-layered low pass filter is designed and simulated using CST software with dielectric constant of 4.4 and substrate height of $1.67 \mathrm{~mm}$ for a cut off frequency of $2.0 \mathrm{GHz}$

Richard's transformation and Kuroda's identities are used to translate a lumped element filter to a transmission line filter. Both identities are used to calculate the impedance of each transmission line segment based on its equivalent lumped element. In order to accommodate for Richard's Transformation and Kuroda's identities, it is essential to control the characteristic impedance of each lumped element or each transmission line segment.

The design of the low-pass micro strip filter having a resonant frequency $2.0 \mathrm{GHz}$ requires a series of steps. In order to design a low-pass filter with such specification it is required to design the filter using electronic circuit theory at normalized values. Further Richard's transformation is used to convert the series inductors to the equivalent series stubs, and the shunt capacitors to the equivalent shunt stubs. The Kuroda's identities are used to transform the series stubs into shunt stubs due to the difficulty of implementing series stubs in microstrip form. The lengths of the shunt stubs are frequency scaled to meet the cut off frequency requirement and the circuit is impedance scaled to 50. Subsequently, the filter's segments are converted into microstrip stubs using microstrip theory. The proposed microstrip filter is then designed and simulated using CST software and fabricated to compare the results.

On top layer a ninth order microstrip low pass filter is designed at a resonant frequency $2.0 \mathrm{GHz}$. Circuit diagram of a low pass filter with lumped element is shown in Fig. 3.

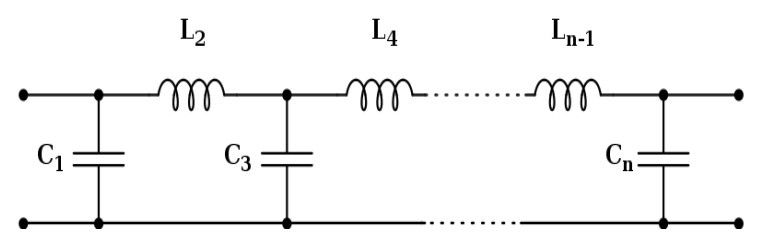

Fig. 3 Circuit diagram of low pass filter

In order to design the microstrip low pass filter with a $3 \mathrm{~dB}$ ripple, the first step is to model the microstrip using electronic circuit theory. The normalized prototype low pass filter values are tabulated in Table 1.

Table 1 Normalized Low Pass Prototype Element Values

\begin{tabular}{|l|l|l|}
\hline g1 & 1.1957 & C1 \\
\hline g2 & 1.1426 & L2 \\
\hline g3 & 2.1346 & C3 \\
\hline g4 & 1.16167 & L4 \\
\hline g5 & 2.2054 & C5 \\
\hline g6 & 1.16167 & L6 \\
\hline g7 & 2.1346 & C7 \\
\hline g8 & 1.1426 & L8 \\
\hline g9 & 1.0 & C9 \\
\hline
\end{tabular}

The lumped values are further converted into distributed elements by using Richard's and Kuroda's identities. The impedance of each stub, excluding the center shunt stub, are multiplied by a factor so that the impedances of the stubs are equivalent to those as shown in Fig. 4.

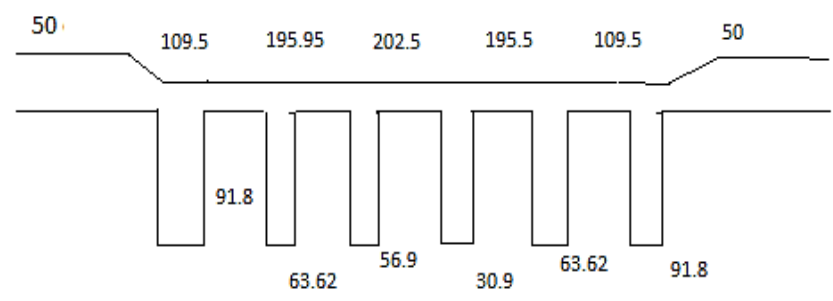

Fig. 4 Low pass filter showing required impedance values

The values of length (l) and width (w) of series and shunt stubs are calculated using the following equations [3]:

For $\mathrm{W} / \mathrm{h} \leq 2$

$$
\frac{W}{h}=\frac{8 \exp (A)}{\exp (2 A)-2}
$$

With

$$
A=\frac{Z_{c}}{60}\left\{\frac{\varepsilon_{r}+1}{2}\right\}^{1.5}+\frac{\bar{\varepsilon}_{r}-1}{\varepsilon_{r}+1}\left\{0.23+\frac{0.11}{\varepsilon_{r}}\right\}
$$


And for $\mathrm{W} / \mathrm{h} \geq 2$

$$
\begin{aligned}
& \frac{W}{h}=\frac{2}{\pi}\left\{(B-1)+\ln (2 \mathrm{~B}-1)+\frac{\varepsilon_{r}-1}{2 \varepsilon_{r}}[\ln (\mathrm{B}-1)+\right. \\
& \left.\left.0.39-\frac{0.61}{s_{r}}\right]\right\}
\end{aligned}
$$

With

$$
B=\frac{60 \pi^{2}}{Z_{c} \sqrt{\epsilon_{r}}}
$$

The calculated values of length and width of the proposed microstrip low pass filter corresponding to their impedances are tabulated in Table II.

Table 2 Calculated Values Of Length And Width

\begin{tabular}{|l|l|l|}
\hline Impedance $(\mathrm{ohm})$ & Length $(\mathrm{mm})$ & Width $(\mathrm{mm})$ \\
\hline 50 & 10.275 & 3.0599 \\
\hline 91.8 & 10.7175 & .890836 \\
\hline 109.75 & 10.85625 & .5416 \\
\hline 63.625 & 9.9675 & 1.9976 \\
\hline 195.95 & 11.2275 & .050929 \\
\hline 56.9 & 10.365 & 2.4524 \\
\hline 202.45 & 11.2425 & .04262 \\
\hline 30.9 & 9.7575 & 9.90406 \\
\hline
\end{tabular}

The proposed low pass filter is designed on top layer using CST software as shown in Fig. 5(a) and hardware design in Fig. 5(b), where the series stub represent inductance and shunt stub represent capacitance.

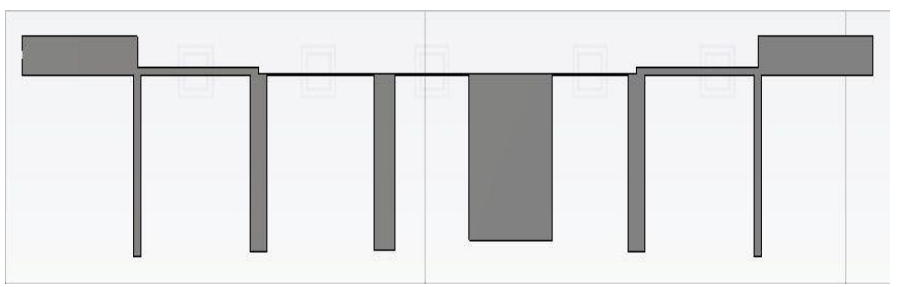

Fig. 5(a) Top layer with proposed low pass filter

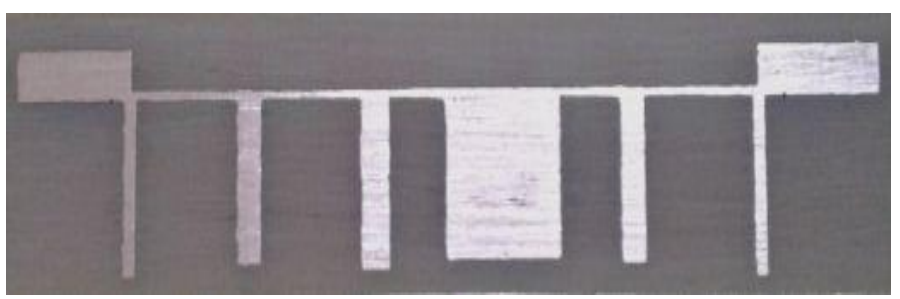

Fig. 5(b) Top layer view of proposed low pass filter

And the CSRR structures are simulated on the middle layer as shown in Fig. 6.

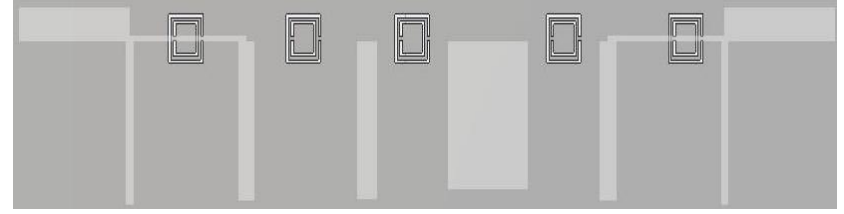

Fig. 6(a) Middle layer with CSRR structure

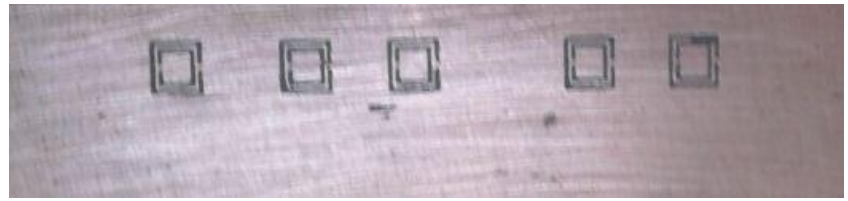

Fig. 6(b) Hardware design of middle layer with CSRR structure

\section{RESULTS AND DISCUSSION}

The design of the multilayered microstrip low pass filter with a resonant frequency $2.0 \mathrm{GHz}$ with low pass structure on the top layer, CSRR on the middle layer and an empty ground layer is designed and simulated.

Two glass epoxy printed circuit boards were patterned, stacked and attached closely for fabrication of the proposed filter. A photograph of the fabricated low pass filter is shown in Fig.7.

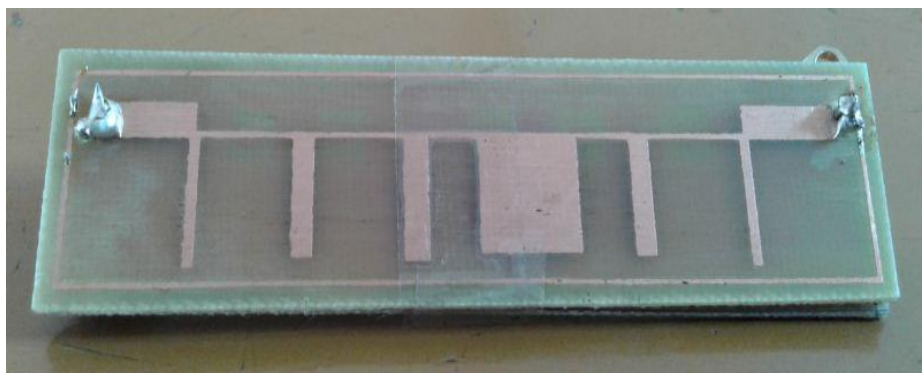

Fig. 7(a) Photograph of fabricated low pass filter (top view)

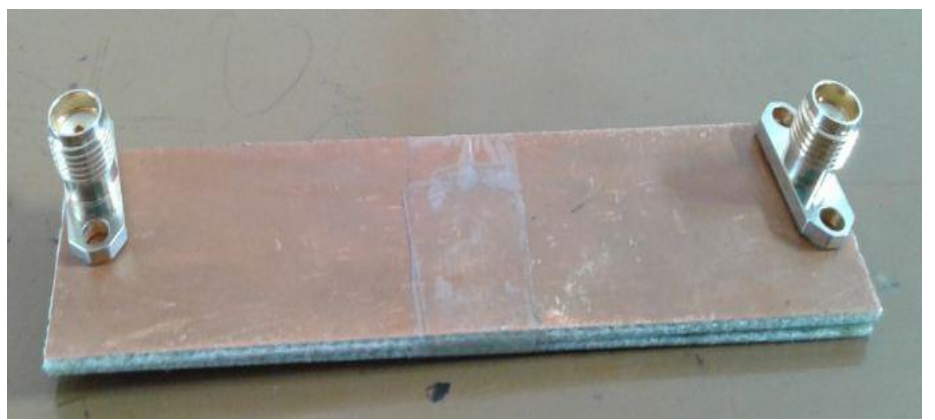

Fig. 7(b) Photograph of fabricated low pass filter (bottom view)

The filter responses obtained by simulating using CST and hardware are in close approximation with each other as shown in Fig. 8. 


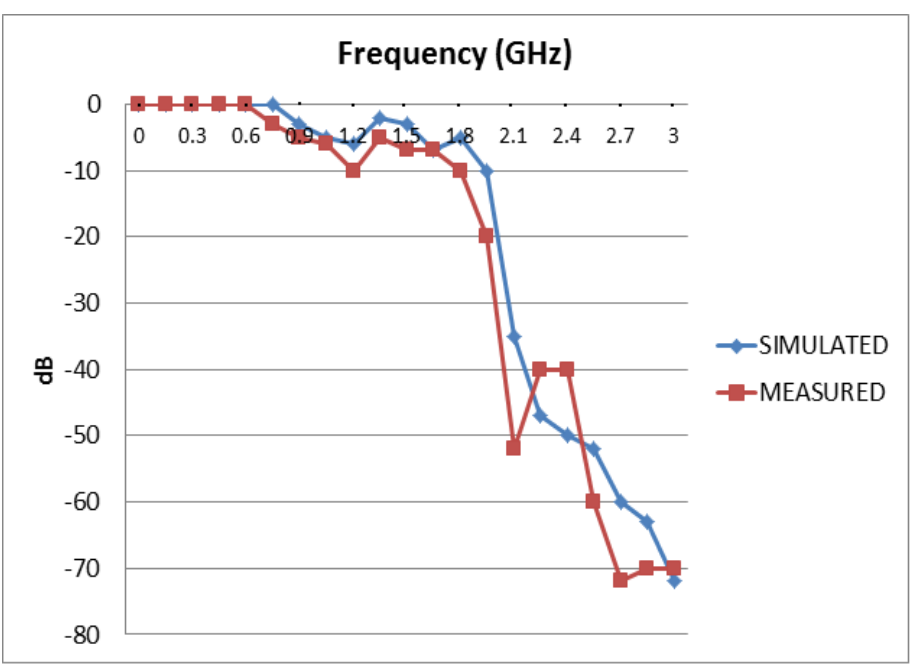

Fig. 8 Response of low pass filter (simulated v/s measured)

From Fig. 8 it can be seen that the filter has a pass band from 0 to $2.0 \mathrm{GHz}$. The filter has approximately zero ripples in pass band and in stop band as suggested by the property of CSRR structures. As can be seen, the simulated and measured results are well in accordance with each other. The measured result shows a gain of $-53 \mathrm{~dB}$ whereas the simulated result shows a gain of $-35 \mathrm{~dB}$. Thus inferring that that the fabricated filter has a better performance as compared to the simulated one. Thus the overall performance of the final design of the multilayered microstrip low pass filter easily met the proposed requirements.

\section{ACKNOWLEDGEMENTS}

The authors would like to thank Professor Dr. P. K. Singhal, department of Electronics and Communication, MITS, Gwalior and Assistant Professor Deepti Gupta, department of electronics and Communication, HCST, Mathura for their helpful discussion.

\section{REFERENCES}

[1] David Pozar, "Microwave Engineering", Third Edition, John Wiley \& Sons, 2005, p.405.

[2] Ali, Z. Hu, "Negative permittivity metamaterial microstrip binomial low pass filter with sharper cut-off and reduced size", 2008.

[3] Jia-Sheng Hong, M. J. Lancaster, "Microstrip Filters for RF/Microwave Applications", Third Edition, John Wiley \& Sons, New York, 2001, p.80-81.

[4] Ying $\mathrm{Xu}$ and Arokiaswami Alphones, "Novel DGSCSRR-based microstrip low pass filter with ultra wide suppression", Int. J. Ultra Wideband Communications and Systems, Vol. 1, No. 3, 2010.

[5] Hon Kuan, Yueh-Ling Lin, Ru-Yuan Yang, Yu-Chi Chang "A Multi-layered parallel coupled microstrip bandpass filter with embedded SIR cells to have a broad upper rejection band", IEEE Microwave and Wireless Components letters, vol. 20, no.1, January 2010.

[6] E. Shamonia, L. Solymar: "Metamaterials: How the subject started" (Metamaterials 1), 2007, p.12-18, p. 615.

[7] M. Gil, J. Bonache, F. Martín: "Metamaterial filters: A review" (Metamaterials 2), 2008, p.186-197.

[8] Xia Wenxiang, Lin Xinfeng, Hu Yongzhong, "Design of Compact Ultra Wide-Stopband Lowpass Filter", 2011.

[9] Abraham Ortega, Leonardo R.A.X. de Menezes, A.J. Martins Soares and Humberto Abdalla Jr., "Design of Low-Pass Microstrip Filters Based on Defected Ground Structure", 2011.

[10] Alexander Miller, Jiasheng Hong, "Cascaded Coupled Line Filter With Reconfigurable Bandwidths Using LCP Multilayer Circuit Technology", IEEE transactions on Microwave Theory and Techniques, vol. 60, no. 6, June 2012.

\section{BIOGRAPHIES}

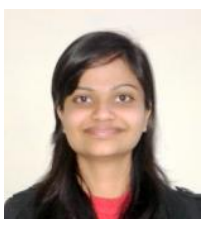

Prachi Agrawal received her B.Tech. Degree from Gautam Buddha Technical University, India, in 2012 And she is currently working toward the M.Tech. Degree in Microwave Engineering from Madhav Institute of Science and Technology, India Her research interest includes the design, development, and modeling of microstrip structures and their applications.

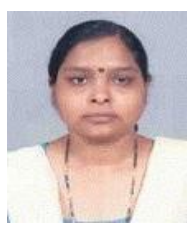

Dr. Vandana V. Thakare received the B.Tech. Degree from Rani Durgawati University, India in 1999 She received her M.Eng. Degree and Ph.D. degree from Rajeev Gandhi Prodyogik Vishwavidhyalaya, India, in 2003 and 2011, respectively She has been into technical teaching for 14 years in the field of Electronics and Communication. She has received National ISTE best women Engineer teacher award in 2011. Her research field includes Microwave Engineering. 